\title{
ON A FREE PISTON PROBLEM FOR STOKES AND NAVIER-STOKES EQUATIONS
}

\author{
Boris MuHa AND Zvonimir TUteK \\ University of Zagreb, Croatia
}

\begin{abstract}
Our goal is to model and analyze a stationary fluid flow through the junction of two pipes in the gravity field. Inside 'vertical' pipe there is a heavy piston which can freely move along the pipe. We are interested in the equilibrium position of the piston in dependence on geometry of junction. Fluid is modeled with the Navier-Stokes equations and the piston is modeled as a rigid body. We formulate corresponding boundary value problem and prove an existence result. The problem is nonlinear even in case of the Stokes equations for fluid flow; we prove non-uniqueness of solutions and illustrate it with some numerical examples. Furthermore, derivation and analysis of the linearized problem are presented.
\end{abstract}

\section{INTRODUCTION}

The piston problem is well known and studied by mathematicians as well as by engineers. It is mainly referred to problems where fluid flow is generated by known motion of the piston. The goal of this paper is to analyze a kind of free boundary problem related to the piston problem. More precisely, we consider fluid flow which is on some part of the boundary delimited by a piston which can move freely along some path. In this paper we restrict ourselves to the stationary case. This problem is also an example of a fluid-rigid body interaction problem. Such problems have been studied intensively in the last decade (see for example $[4,7,10,12]$ and references cited there). However, in these papers rigid body is fully immersed in the fluid, so there is no contact between the rigid body and boundary of the domain filled by fluid. In our case rigid body (the piston) is part of the boundary and therefore different

2010 Mathematics Subject Classification. 35Q30, 76D05, 74F10.

Key words and phrases. Navier-Stokes equations, free piston problem, fluid-rigid body interaction. 
techniques are required. We note that time-dependent free piston problem for gas dynamics have been considered by Takeno ([19]), and D'Acunto and Rionero ([5]). Authors are not aware of any mathematical paper about the free piston problem in case of the Navier-Stokes equations.

In second section we give physical background and precise mathematical formulation of the problem. Furthermore, we give some examples of simple solutions which will provide better understanding of the problem and outline some properties of the solution which are used through this paper. In section three we prove the existence theorems, both for the Stokes and the NavierStokes case. Section four deals with non-uniqueness of solution and we prove occurrence of bifurcation phenomena. In this section we also derive explicit linearized equations which we use in identifying type of bifurcation, but which are also crucial for the numerical analysis of the problem. Main results of the paper are contained in these two sections. In section five we present few simple numerical experiments.

\section{Formulation of the PRoblem}

2.1. Interpretation, geometry and notations. Our goal is to model and analyze flow of Newtonian fluid through system of two pipes with a piston in the "vertical" pipe. More precisely, we consider system of two pipes which consists of horizontal and "vertical" pipe. The angle between horizontal and vertical pipe is measured by $\alpha$; by definition, $\alpha=0$ if the angle between horizontal and vertical pipe is $\pi / 2$. Length of horizontal pipe is $2 l$ and its diameter is $d_{1}$, diameter of "vertical" pipe is $d_{2}$ (see figure 1 ). We consider the problem in a gravity field.

In vertical pipe we have heavy piston which can only move along the "vertical" pipe without rotations. The friction is neglected. In this paper our goal is to determine stationary state of the system, i.e., stationary fluid flow and equilibrium of the piston, and its dependence on geometry, especially on angle $\alpha$. The piston is modeled as a rigid body, thus its motion is given by Newton's second law. Fluid is Newtonian and incompressible so its motion is given by the Navier-Stokes equations. The fluid flow domain is considered as a control volume; we have artificial boundaries as inflow and outflow parts of the boundary on the edges of the horizontal pipe. Coordinate $x_{1}$ is along the horizontal pipe and $x_{3}$ is in the opposite direction of acceleration of the gravity. We assume that flow is driven by known pressure difference on these parts; note that other types of boundary conditions can be imposed.

Let us now introduce some notations and precise assumptions on the geometry. Let $h$ be height of the piston in selected coordinate frame, and let $\Omega_{h}^{\alpha} \subset \mathbb{R}^{3}$ denote domain occupied by the fluid. More precisely, $\Omega_{h}^{\alpha}=$ $\Omega_{1} \cup \Omega_{0} \cup \Omega_{2}^{h}$, where $\Omega_{1}$ and $\Omega_{2}^{h}$ are pipes, i.e., have the form in different 
coordinate frames, possibly non-orthogonal:

$$
\Omega_{i}=\left\{\left(y_{1}, y_{2}, y_{3}\right): 0 \leq y_{1} \leq L_{i},\left(y_{2}, y_{3}\right) \in S_{i}\right\}, \quad S_{i} \subset \mathbb{R}^{2}, i=1,2 .
$$

Only the "vertical" pipe $\Omega_{2}^{h}$ depends on $h$ and $\alpha$. The lower basis of the piston $\Sigma_{h}$ will be considered as a subset of the $x_{3}=$ const. plane. Origin of the coordinate frame is chosen in a such way that the lower end of the "vertical" pipe $\Sigma_{0}$ is a subset of the $x_{3}=0$ plane. We assume that $\Sigma_{0}$ is symmetric w.r.t. $x^{\prime}=\left(x_{2}, x_{3}\right)$-plane. Furthermore, we suppose that $\Omega_{0} \cup \Omega_{1}$ (domain without "vertical" pipe) is symmetric w.r.t. the $x^{\prime}$-plane; we will show that this symmetry assumption is not a restriction. Note that $\Omega_{0}$ is extension of the vertical pipe up to the boundary of $\Omega_{1}$; its shape is complicated in general, in $2 D$ case is an empty set. Inflow and outflow regions are denoted by $\Sigma_{p}$ and $\Sigma_{k}$ respectively. $\Gamma=\partial \Omega_{h}^{\alpha} \backslash\left(\Sigma_{p} \cup \Sigma_{k} \cup \Sigma_{h}\right)$ is a rigid boundary. We only consider $3 D$ case, but formulation of problem in $2 D$ case is straightforward and all results are valid also in this case. Furthermore, all numerical experiments are done in $2 D$ case because of technical simplicity. We suppose that the domain is locally Lipschitzian. Nevertheless, if we want to obtain regularity results we can assume smoothness of the domain by smoothing the angle at the piston boundary.

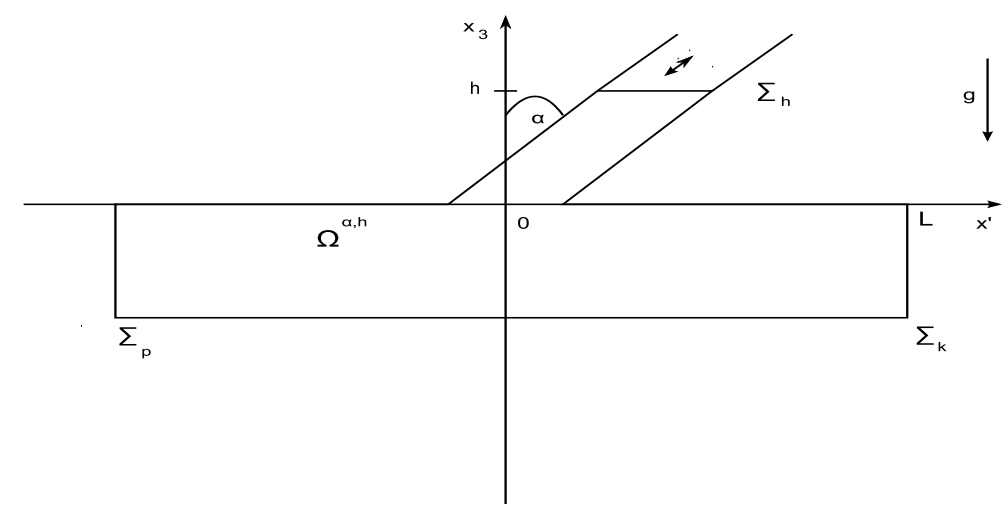

Figure 1. $\Omega_{h}^{\alpha}$

2.2. Mathematical model. Since the fluid is modeled by the Navier-Stokes equations, the stress tensor is given by $T=-p I+2 \mu \operatorname{sym}(\nabla \mathbf{u})$, where $\mathbf{u}$ is velocity of the fluid, $p$ pressure and $\mu$ viscosity. Let $\mathbf{s}=\sin \alpha \mathbf{e}_{1}+\cos \alpha \mathbf{e}_{3}$ be direction of the "vertical" pipe. Total fluid force on the piston in direction $\mathbf{s}$ on height $h$ is given by the formula:

$$
F^{\alpha}(h)=-\int_{\Sigma_{h}} T \mathbf{n} \cdot \mathbf{s},
$$


where $\mathbf{n}$ denotes in general the unit outer normal; here $\mathbf{n}=\mathbf{e}_{3}$. Differential formulation of our problem is: find $(\mathbf{u}, p, h) \in H^{1}\left(\Omega_{h}^{\alpha}\right)^{3} \times L^{2}\left(\Omega_{h}^{\alpha}\right) \times \mathbb{R}_{+}$such that

$$
\begin{gathered}
-\mu \triangle \mathbf{u}+\rho(\nabla \mathbf{u}) \mathbf{u}+\nabla p=-g \rho \mathbf{e}_{3} \quad \text { in } \Omega_{h}^{\alpha}, \\
\operatorname{div} \mathbf{u}=0 \quad \text { in } \Omega_{h}^{\alpha}, \\
\mathbf{u}=0 \quad \text { on } \Gamma, \\
\mathbf{u}=0 \quad \text { on } \Sigma_{h}, \\
\mathbf{u} \times \mathbf{n}=0, p+\frac{1}{2}|\mathbf{u}|^{2}=P_{j}-g \rho x_{3} \quad \text { on } \Sigma_{j}, j=p, k, \\
F^{\alpha}(h)=P_{0} .
\end{gathered}
$$

Here $\rho$ is density of the fluid, $g$ gravity constant and $P_{0}$ a constant that takes into account weight of the piston and atmospheric pressure. First two equations are just the Navier-Stokes equations for motion of an incompressible Newtonian fluid. Boundary conditions $(2.2)_{3}$ and $(2.2)_{4}$ are no slip boundary conditions on the rigid boundary. Condition $(2.2)_{6}$ is balance of forces on the piston. $F^{\alpha}(h)$ is well defined because with our choice of function spaces we have $T \in L^{2}\left(\Omega_{h}^{\alpha}\right)^{3 \times 3}$ and $\operatorname{div} T \in L^{\frac{3}{2}}\left(\Omega_{h}^{\alpha}\right)^{3}$. $P_{p}$ and $P_{k}$ are known constants; since flow is assumed to be driven by the pressure difference on inflow and outflow boundary, we can assume that $P_{p}=-P_{k}$ with possible redefinition of the constant $P_{0}$.

The problem has two non-linearities. One that comes from the NavierStokes equations is classical (see [20]). The second one comes from the fact that domain is unknown and therefore $F$ is a nonlinear function.

REMARK 2.1. Boundary condition $(2.2)_{5}$ were introduced in [3]. For the Stokes case instead of dynamic pressure $p+\frac{1}{2}|\mathbf{u}|^{2}$ we have just pressure $p$. We have also different possibilities of boundary conditions on $\Sigma_{p / k}$. Since we are interested in effects near the junction and the piston, a natural choice would be to prescribe boundary conditions at the infinity. Because we also want to make numerical experiments, we need "artificial" boundary conditions on $\Sigma_{p / k}$. Some of other possible choices are the Dirichlet boundary conditions and "do nothing" boundary conditions; for more details about artificial boundary conditions see [2]. In the present choice of boundary conditions $(2.2)_{5}$ we have fixed pressure on the boundary and there are known regularity results for this type of boundary conditions ([1]). All results of this paper are valid for every choice of 'good' artificial boundary conditions and for boundary conditions imposed at the infinity.

2.3. Examples. First we consider few simple examples for the Stokes case which will illustrate some basic characteristics od problem (2.2).

EXAMPLE 2.2. This example shows why we need to consider the problem in the gravity field. Let $g=0$ and $\alpha=0$, i.e., we consider problem with no gravity and in symmetric domain w.r.t. $x_{3}$-axis. Let $h$ be fixed. 
Then by simple calculation we can verify that the symmetry of domain and boundary data implies the following symmetry of solution of the Stokes problem $(2.2)_{1-5}$ :

$$
\begin{gathered}
u_{x_{1}}^{h}\left(-x_{1}, x^{\prime}\right)=u_{x_{1}}^{h}\left(x_{1}, x^{\prime}\right), u_{x^{\prime}}^{h}\left(-x_{1}, x^{\prime}\right)=-u_{x^{\prime}}^{h}\left(-x_{1}, x^{\prime}\right), \\
p^{h}\left(-x_{1}, x^{\prime}\right)=-p^{h}\left(x_{1}, x^{\prime}\right), x^{\prime}=\left(x_{2}, x_{3}\right) \in \mathbb{R}^{2}, h \in \mathbb{R}_{+} .
\end{gathered}
$$

Further computation gives $F(h)=0$, so in this case problem (2.2) has a solution if and only if $P_{0}=0$, and in this case there are infinitely many solutions.

EXAMPLE 2.3. Let us now consider the same symmetric domain, but this time in the gravity field. Let $p_{H}(x, y)=-\rho g x_{3}$ be the hydrostatic pressure. Let $h \in R_{+}$; by $\left(\mathbf{u}^{h}, q^{h}\right)$ we denote a solution of the Stokes problem in $\Omega_{h}^{0}$ from the previous example, i.e., with $P_{0}=0$. Then $\left(\mathbf{u}^{h}, q^{h}+p_{H}\right)$ is a solution of the non-homogeneous Stokes problem in $\Omega_{h}^{0}$. By straightforward calculation we find that $F(h)=-\rho g h\left|\Sigma_{0}\right|, h \in R_{+}$. We conclude that in this case the whole problem $(2.2)$ has a unique solution $\left(\mathbf{u}^{h}, q^{h}+p_{H}, h\right)$, where $h$ is given by the formula $h=-\frac{P_{0}}{g \rho \mid \Sigma_{0}}$.

REMARK 2.4. Let $\left(\mathbf{u}^{h}, q^{h}+p_{H}\right)$ be the solution of the Stokes problem in domain $\Omega_{h}^{\alpha}$. Using transformation (2.3) we get function $\left(\overline{\mathbf{u}}^{h}, \bar{q}^{h}+p_{H}\right)$ defined on domain $\Omega_{h}^{-\alpha}$. Simple calculation shows that $\left(\overline{\mathbf{u}}^{h}, \bar{q}^{h}+p_{H}\right)$ is the solution of the Stokes problem with the same boundary conditions but in domain $\Omega_{h}^{-\alpha}$. Now from identities $\int_{\Sigma_{h}} q^{h}=-\int_{\Sigma_{h}} \bar{q}^{h}$ and $\int_{\Sigma_{h}} \partial_{x_{3}} u_{x_{1}}^{h}=\int_{\Sigma_{h}} \partial_{x_{3}} \bar{u}_{x_{1}}^{h}$ it follows:

$$
\left(F^{\alpha}(h)+\rho g h\right)=-\left(F^{-\alpha}(h)+\rho g h\right) .
$$

Thus, for $h$ fixed, $F^{\alpha}(h)$ is an odd function of the angle $\alpha$.

\section{EXISTENCE RESULTS}

In this section we derive sufficient condition for problem (2.2) to have at least one solution. In the Navier-Stokes case there will be additional condition on the smallness of data as it is expected. In the sequel index $\alpha$ will be omitted since existence results are proved for arbitrary $\alpha$.

We begin with a simple lemma which describes function $F$ more precisely. Similar result can be found in [14]. Let us define the function space

$$
\mathcal{U}^{h}=\left\{\mathbf{u} \in H^{1}\left(\Omega_{h}\right)^{3}: \operatorname{div} \mathbf{u}=0, \mathbf{u}=0 \text { on } \Gamma \cup \Sigma_{h}, \mathbf{u} \times \mathbf{n}=0 \text { on } \Sigma_{p} \cup \Sigma_{k}\right\} .
$$

LEMMA 3.1. Let $\left(\mathbf{u}^{h}, p^{h}\right) \in \mathcal{U}^{h} \times L^{2}\left(\Omega_{h}\right)$ be a solution of the Navier-Stokes system in $\Omega_{h}$ with boundary conditions as in problem (2.2) ${ }_{1-5}$. Then

$$
F(h)=\cos \alpha \int_{\Sigma_{h}} p^{h}-\mu \sin \alpha \int_{\Sigma_{h}} \partial_{x_{3}} u_{1}^{h}, \quad h \geq 0,
$$

where the integrals are taken in a dual sense. 
Proof. The proof is divided in two steps. First we consider smooth solutions. Because $\mathbf{u}=0$ on $\Sigma_{h}$, simple calculation shows that

$$
\int_{\Sigma_{h}}(\operatorname{sym} \nabla \mathbf{u}) \mathbf{n} \cdot \mathbf{s}=\int_{\Sigma_{h}}\left(\frac{1}{2} \sin \alpha \partial_{x_{3}} u_{1}+\cos \alpha \partial_{x_{3}} u_{3}\right) .
$$

Since solution is smooth, divergence of the velocity is zero up to the boundary, so $\partial_{x_{3}} u_{3}=-\partial_{x_{1}} u_{1}-\partial_{x_{2}} u_{2}$. Hence $\partial_{x_{3}} u_{3}=0$ on $\Sigma_{h}$ and assertion of lemma follows.

If the solution is not smooth, the trace operator for $p^{h} \in L^{2}\left(\Omega_{h}\right)$ is not well defined. Thus we consider the space

$$
\begin{gathered}
\left\{\left(\mathbf{u}^{h}, p^{h}\right) \in \mathcal{U}^{h} \times L^{2}\left(\Omega_{h}\right):\right. \\
: T\left(\mathbf{u}^{h}, p^{h}\right)=-p I+\mu \operatorname{sym}(\nabla \mathbf{u}) \in L^{2}\left(\Omega_{h}\right), \\
\left.\operatorname{div} T \in L^{3 / 2}\left(\Omega^{h}\right)\right\} .
\end{gathered}
$$

Note that choice $\operatorname{div} T \in L^{3 / 2}\left(\Omega^{h}\right)$ covers both, Stokes and Navier-Stokes case. For such space there exists normal trace operator $T \mapsto T \mathbf{n}_{\mid \Sigma_{h}}$. From the first part of proof we know that for smooth function $T \mathbf{n} \cdot \mathbf{s}=\cos \alpha p-\mu \sin \alpha \partial_{x_{3}} u_{x_{1}}^{h}$ on $\Sigma_{h}$ in a trace sense. Therefore by extension, formula (3.1) holds.

3.1. Stokes flow. First we consider the Stokes flow, i.e., motion of the fluid is described by the Stokes equations:

find $(\mathbf{u}, p, h) \in H^{1}\left(\Omega_{h}^{\alpha}\right)^{3} \times L^{2}\left(\Omega_{h}^{\alpha}\right) \times \mathbb{R}_{+}$such that

$$
\begin{gathered}
-\mu \triangle \mathbf{u}+\nabla p=-g \rho \mathbf{e}_{3} \quad \text { in } \Omega_{h}^{\alpha}, \\
\operatorname{div} \mathbf{u}=0 \quad \text { in } \Omega_{h}^{\alpha}, \\
\mathbf{u}=0 \quad \text { on } \Gamma, \\
\mathbf{u}=0 \quad \text { on } \Sigma_{h}, \\
\mathbf{u} \times \mathbf{n}=0, p=P_{j}-g \rho x_{3}, \quad \text { on } \Sigma_{j}, j=p, k . \\
F(h)=P_{0},
\end{gathered}
$$

Theorem 3.2. There exists $P \in \mathbb{R}$ such that for every $P_{0} \leq P$ problem (3.2) has at least one solution.

Proof. Restriction on $P_{0}$ is expected, since fluid can not 'support' too heavy piston.

Since for every fixed $h$ existence of corresponding $\left(\mathbf{u}^{h}, p^{h}\right)$ is well known, it is enough to prove that there exists $h$ such that $F(h)=P_{0}$ for $P_{0}$ small enough. Furthermore, $F$ is continuous function because of continuous dependence of solutions of the Stokes equations on change of a domain (see for example [17]) and because of continuity of the trace operator.

We will prove that $\lim _{h \rightarrow \infty} F(h)=-\infty$ and the assertion of theorem follows directly. For fixed $h$ solution can be written in the form $\left(\mathbf{u}^{h}, q^{h}+p_{H}\right)$, where $\left(\mathbf{u}^{h}, q^{h}\right)$ is solution of the homogeneous Stokes equation and $p_{H}$ is the hydrostatic pressure. Because of $\lim _{h \rightarrow \infty} \int_{\Sigma_{h}} p_{H}=-\infty$, it is enough to prove boundedness of $\int_{\Sigma_{h}} q^{h}$ and $\int_{\Sigma_{h}} \partial_{x_{3}} u_{x_{1}}^{h}$ in $h$, see Lemma 3.1 We estimate 
these integrals using Leray's problem. Let $\Omega_{\infty}$ be domain with unbounded "vertical" pipe, i.e., $\Omega_{\infty}=\cup_{h>0} \Omega_{h}$. Now let us consider Leray's problem in $\Omega_{\infty}$ with prescribed flux zero at infinity; this is equivalent to $\mathbf{v}(\mathbf{x}) \rightarrow 0$, $|\mathbf{x}| \rightarrow \infty$.

$$
\begin{gathered}
-\mu \Delta \mathbf{v}+\nabla \tau=0 \text { in } \Omega_{\infty}, \\
\operatorname{div} \mathbf{v}=0 \text { in } \Omega_{\infty}, \\
\mathbf{v}=0 \text { on } \Gamma_{\infty}, \\
\mathbf{v} \rightarrow 0, x \in \Omega_{\infty},|x| \rightarrow \infty, \\
\mathbf{v} \times \mathbf{n}=0, \tau=P_{j}, \quad \text { on } \Sigma_{j}, j=p, k .
\end{gathered}
$$

Note that in our original problem flux is zero in vertical pipe for each $h$, so it is natural to assume flux zero at infinity for the Leray problem. Existence of solution of this problem and its asymptotic behavior is well known, see [8]. Proof of the theorem will be complete when we prove the following lemma.

LEMMA 3.3. Let $(\mathbf{v}, \tau)$ be the solution of Leray's problem (3.3) and let $\left(\mathbf{u}^{h}, q^{h}+p_{H}\right)$ be the solution of the corresponding Stokes problem in $\Omega_{h}, h>0$. Then:

$$
\left\|\mathbf{u}^{h}-\mathbf{v}\right\|_{H^{1}\left(\Omega^{h}\right)}+\left\|q_{h}-\tau\right\|_{L^{2}\left(\Omega_{h}\right)} \rightarrow 0 \text { as } h \rightarrow \infty .
$$

Proof. Let us define auxiliary functions $\mathbf{w}_{h}=\mathbf{u}_{h}-\mathbf{v}$ and $r_{h}=p_{h}-\tau$ on $\Omega_{h}$. These functions are solutions of the following problem:

$$
\begin{gathered}
-\mu \triangle \mathbf{w}_{h}+\nabla r_{h}=0 \text { in } \Omega_{h}, \\
\operatorname{div} \mathbf{w}_{h}=0 \text { in } \Omega_{h}, \\
\mathbf{w}_{h}=0 \text { on } \Gamma, \\
\mathbf{w}_{h}=-\mathbf{v} \text { on } \Sigma_{h}, \\
\mathbf{w}_{h} \times \mathbf{n}=0, r_{h}=0 \text { on } \Sigma_{j}, j=p, k .
\end{gathered}
$$

It is classical that

$$
\left\|\mathbf{w}_{h}\right\|_{H^{1}\left(\Omega_{h}\right)}+\left\|r_{h}\right\|_{L^{2}\left(\Omega_{h}\right)} \leq C_{h}\|\mathbf{v}\|_{H^{\frac{1}{2}}\left(\Sigma_{h}\right)}
$$

It is well known that $\mathbf{v}$ and all its derivatives decays to 0 uniformly with exponential speed. Assertion of lemma now follows from the fact that $C_{h}$ in fact does not depend on $h$; this can be proved by homogenization of boundary condition on $\Sigma_{h}$ which will be done explicitly in the Navier-Stokes case.

REMARK 3.4. In the case of regular domain, we have regular solution and also convergence of all derivatives. 
3.2. Navier-Stokes flow. The main idea of the proof of existence of solution for the Navier-Stokes case is analogous. We just need to prove Lemma 3.3 for the Navier-Stokes equations. Since we are now dealing with Navier Stokes equations, we will need to restrict ourselves to small data to get estimates which are essential for our proof. From now on we assume that the data are small enough to ensure the uniqueness and existence of solution of Leray's problem for the Navier-Stokes equations in $\Omega_{\infty}$, see [9] for example.

Let $(\mathbf{v}, \tau)$ be the solution of nonlinear Leray's problem in $\Omega_{\infty}$ and let $h>1$. Furthermore, let $\mathbf{V}_{h} \in H^{1}\left(\Omega_{h}\right)$ be a function that satisfies:

$$
\operatorname{div} \mathbf{V}_{h}=0 \text {, }
$$

$\mathbf{V}_{h}=\mathbf{v}$ on $\Sigma_{h}, \mathbf{V}_{h}=0$ on $\partial \Omega_{h} \backslash \Sigma_{h}, \mathbf{V}_{h}\left(x_{1}, x_{2}, x_{3}\right)=0, x_{3}<h-1$.

We know that such function exists because flux is zero in the "vertical" pipe (see [18]). Now, $\mathbf{v}_{h}=\mathbf{v}-\mathbf{V}_{h}$ satisfies the equations:

$$
\begin{gathered}
-\mu \Delta \mathbf{v}_{h}+\rho\left(\nabla \mathbf{v}_{h}\right) \mathbf{v}_{h}+\nabla p_{h}=\mathbf{f}_{h} \quad \text { in } \Omega_{h}, \\
\operatorname{div} \mathbf{v}_{h}=0 \quad \text { in } \Omega_{h}, \\
\mathbf{v}_{h}=0 \quad \text { on } \Gamma, \\
\mathbf{v}_{h}=0 \quad \text { on } \Sigma_{h}, \\
\mathbf{v}_{h} \times \mathbf{n}=0, p_{h}+\frac{1}{2}\left|\mathbf{v}_{h}\right|^{2}=P_{j} \quad \text { on } \Sigma_{j}, j=p, k,
\end{gathered}
$$

where $\mathbf{f}_{h}=-\mu \triangle \mathbf{V}_{h}+(\nabla \mathbf{v}) \mathbf{V}_{h}+\left(\nabla \mathbf{V}_{h}\right) \mathbf{v}-\left(\nabla \mathbf{V}_{h}\right) \mathbf{v}_{h}$. Function $\mathbf{V}_{h}$ has the support only in some neighborhood of $\Sigma_{h}$ by definition, so $\mathbf{f}_{h}$ has the same property. From the decay property of Leray's flow it follows that $\left\|\mathbf{f}_{h}\right\|_{H^{-1}\left(\Omega_{h}\right)} \rightarrow 0$ as $h \rightarrow \infty$.

Let us define $\mathbf{w}_{h}=\mathbf{u}_{h}-\mathbf{v}_{h}$ and $r_{h}=p_{h}-\tau$. Now we can subtract weak formulation of problem (3.5) (see [3])

$\left.\mu \int_{\Omega_{h}} \nabla \times \mathbf{v}_{h} \cdot \nabla \times \mathbf{v}+\int_{\Omega_{h}}\left(\left(\nabla \times \mathbf{v}_{h}\right) \times \mathbf{v}_{h}\right)\right) \cdot \mathbf{v}=\left\langle f_{h}, \mathbf{v}\right\rangle-P_{p} \int_{\Sigma_{p}} v_{x_{1}}+P_{k} \int_{\Sigma_{k}} v_{x_{1}}$,

from analogous weak formulation of problem for $\mathbf{u}_{h}$ and take $\mathbf{w}_{h}$ for test function. We get

$$
\left.C\left\|\mathbf{w}_{h}\right\|_{H^{1}\left(\Omega_{h}\right)}^{2} \leq-\int_{\Omega_{h}}\left(\left(\nabla \times \mathbf{w}_{h}\right) \times \mathbf{v}_{h}\right)\right) \cdot \mathbf{w}_{h}+\left\langle f_{h}, \mathbf{w}_{h}\right\rangle ;
$$

here $\langle\cdot, \cdot\rangle$ stands for duality pairing. Finally we have:

$$
\left\|\mathbf{w}_{h}\right\|_{H^{1}\left(\Omega_{h}\right)}+\left\|r_{h}\right\|_{L^{2}\left(\Omega_{h}\right)} \rightarrow 0 \text { as } h \rightarrow \infty .
$$

Notice that this estimate is sufficient for the proof existence theorem. If we want estimates in $H^{m}$ norms in the case of smooth domain, we can proceed in a classical way to obtain them. Therefore we have proved:

TheOREM 3.5. For the data small enough, there exists $P \in \mathbb{R}$ such that for every $P_{0} \leq P$ problem (2.2) has at least one solution. 
REMARK 3.6. In the proof of existence theorem we have used only one geometrical assumption, the one that we have "vertical" pipe. Completely analogous proof holds for infinite horizontal pipe. Furthermore, we have not used symmetry assumption, so theorem holds also for non-symmetric domains. We even have not used assumption that the lower part of domain is a pipe, so proof can be adapted for more general geometry and boundary conditions.

\section{NON-UNIQUENESS OF SOLUTION}

Now we turn to the question of uniqueness of the solution of problem (3.2). We first show that one can not expect uniqueness.

REMARK 4.1. Non-uniqueness of the solution of problem (3.2). Let us take $\alpha \neq 0$ and denote temporarily the total force on $\Sigma_{h}$ by $F^{\alpha}$. We separate the total force on $\Sigma_{h}$ into the part that comes from the hydrostatic pressure and the part that comes from velocity and pressure, i.e., $F^{\alpha}=F_{p}^{\alpha}-$ $\rho g h\left|\Sigma_{h}\right|$. From Remark 2.4 we know that $F_{p}^{\alpha}=-F_{p}^{-\alpha}$ for the same $h$. Assume that function $F_{p}^{\alpha}$ is differentiable (this will be proved later on) and let $\varepsilon>0$ be such that $\frac{d}{d h} F_{p}^{\alpha}(\varepsilon) \neq 0$. We assume $\frac{d}{d h} F_{p}^{\alpha}(\varepsilon)>0$; we can do that because in the opposite case we just consider $-\alpha$ instead of $\alpha$. Let $\left(\mathbf{u}_{\varepsilon}^{\alpha}, p_{\varepsilon}^{\alpha}-\rho g x_{3}\right)$ be the solution of the Stokes system in $\Omega_{\varepsilon}^{\alpha}$ associated to total force $F^{\alpha}(\varepsilon)$. Because of linearity of the Stokes system, for every $\lambda>0$ the pair $\left(\lambda \mathbf{u}_{\varepsilon}^{\alpha}, \lambda p_{\varepsilon}^{\alpha}-\rho g x_{3}\right)$ is also the solution of the Stokes system in $\Omega_{\varepsilon}^{\alpha}$ with different boundary conditions on $\Sigma_{p / k}$; let us denote the total force associated to this solution by $F_{p, \lambda}^{\alpha}$. Now if we take $\lambda$ large enough, we get that $\frac{d}{d h} F_{p, \lambda}^{\alpha}(\varepsilon)=\lambda \frac{d}{d h} F_{p}^{\alpha}-\rho g h\left|\Sigma_{h}\right|>0$. Since we know that $\lim _{h \rightarrow \infty} \frac{d}{d h} F_{p, \lambda}^{\alpha}(h)=-\rho g\left|\Sigma_{h}\right|$, we conclude that $F_{p, \lambda}^{\alpha}$ is not monotone and therefore there exists $P_{0}$ for which we have at least two stationary states.

Of course, if data are small enough, force from the hydrostatic pressure will be dominant and we will have the unique stationary state. However, as we have seen in example 2.2 , non-uniqueness is not only due to the hydrostatic pressure. Later we will illustrate that with a numerical experiment. Now we want to prove non-uniqueness in a rigorous way and describe it more precisely. In order to achieve that, first we need to derive and analyze the linearized problem. Since the non-linearity is non explicit and hidden in domain $\Omega_{h}^{\alpha}$, first we rewrite our problem in such a way that nonlinearity would be expressed explicitly. We can do that by transforming original problem onto the fixed domain $\Omega:=\Omega_{1}^{0}$ using some change of variables. We use the transformation

$$
\begin{gathered}
\beta_{h}^{\alpha}: \Omega \mapsto \Omega_{h}^{\alpha}, \alpha \in(-\pi / 2, \pi / 2), h>0: \\
\beta_{h}^{\alpha}\left(x_{1}, x_{2}, x_{3}\right)=\left\{\begin{array}{cc}
\left(x_{1}, x_{2}, x_{3}\right), & x_{3}<0, \\
\left(x_{1}+h x_{3} \tan \alpha, x_{2}, h x_{3}\right), & x_{3}>0 .
\end{array}\right.
\end{gathered}
$$


This change of variables has $W^{1, \infty}$ regularity which is enough for the weak formulation of the Stokes problem in $\Omega_{h}^{\alpha}$. Nevertheless, one should notice that $\beta_{h}^{\alpha}$ is not a $C^{1}$ function only on the lower dimensional set $\Sigma_{0}$. One could also work with smooth change of variables, but we prefer this simplest change of variables which gives us the linearized problem explicitly; an explicit formulation of the linearized problem is needed for numerical experiments as well.

We introduce notations: $\Omega_{+}=\Omega \cap\left\{x_{3}>0\right\}$ and $\Omega^{-}=\Omega \cap\left\{x_{3}<0\right\}$ and the function space:

$$
\mathcal{U}=\left\{\mathbf{u} \in H^{1}(\Omega)^{3}: \mathbf{u}=0 \text { on } \Gamma \cup \Sigma, \mathbf{u} \times \mathbf{n}=0 \text { on } \Sigma_{p / k}\right\},
$$

where $\Gamma=\Gamma_{1}^{0}$ and $\Sigma=\Sigma_{1}$. We introduce new unknowns $\mathbf{u}(h, \alpha)$ and $p(h, \alpha)$ by $\mathbf{u}(h, \alpha)=\mathbf{u}_{h}^{\alpha} \circ \beta_{h}^{\alpha}$ and $p(h, \alpha)=p_{h}^{\alpha} \circ \beta_{h}^{\alpha}$. Then by change of variables $\beta_{h}^{\alpha}$ problem (3.2) is transformed to: find $(\mathbf{u}(h, \alpha), p(h, \alpha), h) \in \mathcal{U} \times L^{2}(\Omega) \times \mathbb{R}_{+}$ such that

$$
\begin{aligned}
& h\left(\mu \int_{\Omega^{+}} \nabla^{h, \alpha} \mathbf{u}(h, \alpha) \cdot \nabla^{h, \alpha} \mathbf{v}-\int_{\Omega^{+}} p(h, \alpha) \operatorname{div}^{h, \alpha} \mathbf{v}+\rho g \int_{\Omega^{+}} v_{x_{3}}\right) \\
& +\mu \int_{\Omega^{-}} \nabla \mathbf{u}(h, \alpha) \cdot \nabla \mathbf{v}-\int_{\Omega^{-}} p(h, \alpha) \operatorname{div} \mathbf{v}+\rho g \int_{\Omega^{-}} v_{x_{3}} \\
& =\left\langle G\left(P_{p}, P_{k}\right), \mathbf{v}\right\rangle, \quad v \in \mathcal{U}, \\
& h \int_{\Omega^{+}} q \operatorname{div}^{h, \alpha} \mathbf{u}(h, \alpha)+\int_{\Omega^{-}} q \operatorname{div} \mathbf{u}(h, \alpha)=0, \quad q \in L^{2}(\Omega), \\
& \cos \alpha \int_{\Sigma} p(h, \alpha)-\mu \sin \alpha \int_{\Sigma} \frac{1}{h} \partial_{x_{3}} u_{1}(h, \alpha)-P_{0}=0
\end{aligned}
$$

where $\left\langle G\left(P_{p}, P_{k}\right), \mathbf{v}\right\rangle=\int_{\Sigma_{p}} P_{p} v_{x_{1}}-\int_{\Sigma_{k}} P_{k} v_{x_{1}}$ and

$$
\nabla^{h, \alpha}=\left\{\begin{array}{c}
\nabla \\
\partial_{x_{1}} \\
\partial_{x_{2}}<0, \\
\left(\begin{array}{c}
x_{3}<\frac{1}{h} \partial_{x_{3}}
\end{array}\right) \quad x_{3}>0,
\end{array} \quad \text { and } \quad \operatorname{div}^{h, \alpha}=\nabla^{h, \alpha}\right.
$$

Now we need to specify function spaces on which we consider our non-linear operator. More than $L^{2}$ regularity for pressure is needed for $(4.2)_{3}$ to make sense. On the other hand, due to the lack of regularity of change of variables one can not expect the global regularity. In Lemma 3.1 we have overcome this difficulty by considering $\operatorname{div} T$ which was is some $L^{r}$ space, so trace operator was well defined. Now we can not proceed in analogous way because operator div is transformed into $\operatorname{div}^{h, \alpha}$ and $h$ is also unknown of the problem. Therefore in this section we use regularity results for polyhedral domains proved by Maz'ya and Rossmann ([15]) and introduce some non-standard 
function spaces:

$$
\begin{aligned}
& \mathcal{V}=\left\{\mathbf{v} \in \mathcal{U}: \mathbf{v} \chi_{\mid \Omega_{+}} \in W^{2,8 / 7}\left(\Omega_{+}\right), \mathbf{v} \chi_{\mid \Omega_{-}} \in W^{2,8 / 7}\left(\Omega_{-}\right)\right\}, \\
& \mathcal{Q}=\left\{q \in L^{2}(\Omega): q \chi_{\mid \Omega_{+}} \in W^{1,8 / 7}\left(\Omega_{+}\right), q \chi_{\mid \Omega_{+}} \in W^{1,8 / 7}\left(\Omega_{-}\right)\right\},
\end{aligned}
$$

where $W^{s, p}$ is the standard Sobolev space and $\chi_{\mid A}$ is the characteristic function of set $A$. Let us define $J: \mathbb{R}^{2} \times W^{1 / 8,8 / 7}\left(\Sigma_{0}\right) \rightarrow \mathcal{U}^{\prime}\left(\mathcal{U}^{\prime}\right.$ is the dual space of $\mathcal{U}$ ) by

$$
\left\langle J\left(P_{p}, P_{k}, b\right), \mathbf{v}\right\rangle=\left\langle G\left(P_{p}, P_{k}\right), \mathbf{v}\right\rangle+\int_{\Sigma_{0}} b \mathbf{v} \cdot \mathbf{n}, \quad \mathbf{v} \in \mathcal{U}
$$

We have $\operatorname{supp}\left(J\left(P_{p}, P_{k}, b\right)\right) \subset \Sigma_{p} \cup \Sigma_{k} \cup \Sigma_{0}$. We also need function space:

$$
\begin{gathered}
\mathcal{W}=\left\{F \in \mathcal{U}^{\prime}: F=f+J\left(P_{p}, P_{k}, b\right), f \in L^{8 / 7}(\Omega) \cap \mathcal{U}^{\prime},\right. \\
\left.P_{p}, P_{k} \in \mathbb{R}, b \in W^{1 / 8,8 / 7}\left(\Sigma_{0}\right)\right\} .
\end{gathered}
$$

$\mathcal{V}, \mathcal{Q}$ and $\mathcal{W}$ are Banach spaces with norms

$$
\begin{gathered}
\|\cdot\|_{\mathcal{V}}=\|\cdot\|_{H^{1}(\Omega)}+\|\cdot\|_{W^{2,8 / 7}\left(\Omega_{+}\right)}+\|\cdot\|_{W^{2,8 / 7}\left(\Omega_{-}\right)}, \\
\|\cdot\|_{\mathcal{Q}}=\|\cdot\|_{L^{2}(\Omega)}+\|\cdot\|_{W^{1,8 / 7}\left(\Omega_{+}\right)}+\|\cdot\|_{W^{1,8 / 7}\left(\Omega_{-}\right)}, \\
\left\|f+J\left(P_{p}, P_{k}, b\right)\right\|_{\mathcal{W}} \\
=\|f\|_{L^{8 / 7}(\Omega)}+\left|P_{p}\right|+\left|P_{k}\right|+\|b\|_{W^{1 / 8,8 / 7}\left(\Sigma_{0}\right)}+\left\|f+J\left(P_{p}, P_{k}, b\right)\right\|_{H^{-1}(\Omega)} .
\end{gathered}
$$

Let $\mathbf{S}: \mathcal{V} \times \mathcal{Q} \times \mathbb{R}_{+} \rightarrow \mathcal{W} \times \mathcal{Q} \times \mathbb{R}$ be operator defined by the left hand side of (4.2); $P_{0}$ and $\alpha$ are considered as parameters and in this subsection will be omitted. Notice that function spaces are chosen in a such way that contain all solutions of the problem (4.2) and that trace operator $(4.2)_{3}$ is well defined. Furthermore we can see that space $\mathcal{W}$ is chosen to be the range of the Stokes-like operator on $\mathcal{V}$ and $\mathcal{Q}$. Since $\mathbf{S}$ is linear in first two arguments, we define operator $\mathcal{S}: \mathbb{R}_{+} \rightarrow \mathcal{L}(\mathcal{V} \times \mathcal{Q}, \mathcal{W} \times \mathcal{Q} \times \mathbb{R})$ with

$$
\mathcal{S}(h)(\mathbf{v}, q)=\mathbf{S}(\mathbf{v}, q, h) .
$$

Now we formally calculate the Frechet derivative of $\mathbf{S}$ :

$$
\begin{aligned}
\mathbf{S}\left(\mathbf{u}_{0}\right. & \left.+\varepsilon \mathbf{u}, p_{0}+\varepsilon p, h_{0}+\varepsilon h\right)=\mathcal{S}\left(h_{0}+\varepsilon h\right)\left(\mathbf{u}_{0}+\varepsilon \mathbf{u}, p_{0}+\varepsilon p\right) \\
& =\left(\mathcal{S}\left(h_{0}\right)+\varepsilon h \mathcal{S}^{\prime}\left(h_{0}\right)+o\left(\varepsilon^{2}\right)\right)\left(\mathbf{u}_{0}+\varepsilon \mathbf{u}, p_{0}+\varepsilon p\right) \\
& =\mathcal{S}\left(h_{0}\right)\left(\mathbf{u}_{0}, p_{0}\right)+\varepsilon\left(\mathcal{S}\left(h_{0}\right)(\mathbf{u}, p)+h \mathcal{S}^{\prime}\left(h_{0}\right)\left(\mathbf{u}_{0}, p_{0}\right)\right)+o\left(\varepsilon^{2}\right) .
\end{aligned}
$$

So $\mathbf{S}$ is differentiable if and only if $\mathcal{S}$ is differentiable and then we have:

$$
\mathbf{S}^{\prime}\left(\mathbf{u}_{0}, p_{0}, h_{0}\right)(\mathbf{u}, p, h)=\mathcal{S}\left(h_{0}\right)(\mathbf{u}, p)+h \mathcal{S}^{\prime}\left(h_{0}\right)\left(\mathbf{u}_{0}, p_{0}\right) .
$$

Therefore for formulating the linearized problem it is enough to calculate $\mathcal{S}^{\prime}\left(h_{0}\right)$. Let us consider a stationary state $\left(\mathbf{u}_{0}, p_{0}, h_{0}\right)$, i.e., $\mathbf{S}\left(\mathbf{u}_{0}, p_{0}, h_{0}\right)=0$, 
and insert $\mathbf{u}_{0}+\varepsilon \mathbf{u}, p_{0}+\varepsilon p$ in equation (4.2). By using equalities

$$
\begin{aligned}
& \frac{1}{h_{0}+\varepsilon h}=\frac{1}{h_{0}}-\frac{1}{h_{0}^{2}} \varepsilon h+o\left(\varepsilon^{2}\right), \\
& \nabla^{h_{0}+\varepsilon h, \alpha}=\nabla^{h_{0}, \alpha}-\varepsilon \frac{h}{h_{0}^{2}}\left(\begin{array}{c}
\mathbf{0} \\
\partial_{x_{3}}
\end{array}\right)+o\left(\varepsilon^{2}\right), \quad x_{3}>0,
\end{aligned}
$$

and collecting members of order $o\left(\varepsilon^{2}\right)$, we get

$$
\begin{aligned}
& \left\langle\mathcal{S}_{1}^{\prime}\left(h_{0}\right)\left(\mathbf{u}_{0}, p_{0}\right), \mathbf{v}\right\rangle=\int_{\Omega^{+}}\left(\mu \nabla^{h_{0}, \alpha} \mathbf{u}_{0} \cdot \nabla^{h_{0}, \alpha} \mathbf{v}-\frac{\mu}{h_{0}}\left(\begin{array}{c}
\mathbf{0} \\
\partial_{x_{3}}
\end{array}\right) \mathbf{u}_{0} \cdot \nabla^{h_{0}, \alpha} \mathbf{v}\right. \\
& \left.-\frac{\mu}{h_{0}} \nabla^{h_{0}, \alpha} \mathbf{u}_{0} \cdot\left(\begin{array}{c}
\mathbf{0} \\
\partial_{x_{3}}
\end{array}\right) \mathbf{v}-p_{0} \nabla^{h_{0}, \alpha} \cdot \mathbf{v}+\frac{1}{h_{0}} p_{0}\left(\begin{array}{c}
\mathbf{0} \\
\partial_{x_{3}}
\end{array}\right) \cdot \mathbf{v}+h_{0} g v_{x_{3}}\right) \\
& \mathcal{S}_{2}^{\prime}\left(h_{0}\right)\left(\mathbf{u}_{0}, p_{0}\right)=-\frac{1}{h_{0}^{2}} \partial_{x_{3}}\left(u_{0}\right)_{x_{3}} \\
& \mathcal{S}_{3}^{\prime}\left(h_{0}\right)\left(\mathbf{u}_{0}, p_{0}\right)=\mu \frac{\sin \alpha}{h_{0}^{2}} \int_{\Sigma} \partial_{x_{3}}\left(u_{0}\right)_{x_{1}} .
\end{aligned}
$$

By iterating this procedure we can prove:

Proposition 4.2. $\mathcal{S} \in C^{\infty}\left(\mathbb{R}_{+} ; \mathcal{L}(\mathcal{V} \times \mathcal{Q}, \mathcal{W} \times \mathcal{Q} \times \mathbb{R})\right)$ and $\mathcal{S}^{\prime}$ is given by (4.4). $\mathbf{S} \in C^{\infty}\left(\mathcal{V} \times \mathcal{Q} \times \mathbb{R}_{+} ; \mathcal{W} \times \mathcal{Q} \times \mathbb{R}\right)$ and $\mathbf{S}^{\prime}$ is given by (4.3).

4.1. Bifurcations. With our new notations we may rewrite problem (3.2) (or equivalent problem (4.2)) in the following way:

$$
\text { find }(\mathbf{u}, p, h) \in \mathcal{V} \times \mathcal{Q} \times \mathbb{R} \text { such that } \mathbf{S}\left(\mathbf{u}, p, h ; P_{0}, \alpha\right)=0 \text {. }
$$

As we have seen in Remark 4.1, one can not expect unique stationary state for some boundary data, $\alpha$ and $P_{0}$. Our goal is to identify exact type of non-uniqueness (bifurcation) in dependence of $P_{0}$. Necessary condition for bifurcation point is non-regularity of $\mathbf{S}^{\prime}$ because in opposite case we would have local uniqueness by the implicit function theorem ([13]). Therefore we must consider the linearized problem. Let $\left(\mathbf{u}_{0}, p_{0}, h_{0}\right)$ be some stationary state. We prove some properties of the operator $\mathbf{S}^{\prime}\left(\mathbf{u}_{0}, p_{0}, h_{0}\right)$.

LEMMA 4.3. Operator $\overline{\mathcal{S}}(h)=\left(\mathcal{S}_{1}(h), \mathcal{S}_{2}(h)\right)=\left(\mathcal{S}_{x_{1}}(h), \mathcal{S}_{x^{\prime}}(h), \mathcal{S}_{2}(h)\right) \in$ $\mathcal{L}(\mathcal{V} \times \mathcal{Q}, \mathcal{W} \times \mathcal{Q})$ is a regular linear operator with bounded inverse.

Proof. Notice that $\overline{\mathcal{S}}(h)$ is just a Stokes-like operator in new coordinates. By using inverse transformation we can transform equations back to original domain and solve the Stokes problem with new right-hand side. Then the only nontrivial part is to prove that operator is surjective. Existence of $H^{1} \times L^{2}$ solution verifying given boundary conditions is proved in [3], let us denote it with $(\mathbf{u}, p)$. It remains to prove suitable regularity. We can divide this problem into two Stokes problems in $\Omega_{+}^{h}$ and $\Omega_{-}$which are coupled on $\Sigma_{0}$. 
More precisely, let $\left(\mathbf{u}^{+}, p^{+}\right)$and $\left(\mathbf{u}^{-}, p^{-}\right)$be restrictions of $(\mathbf{u}, p)$ on $\Omega_{+}^{h}$ and $\Omega_{-}$respectively. Then these functions satisfy equations

$$
\begin{array}{cc}
-\mu \triangle \mathbf{u}^{+}+\nabla p^{+}=\mathbf{f}^{+} \text {in } \Omega_{+}^{h}, & -\mu \triangle \mathbf{u}^{-}+\nabla p^{-}=\mathbf{f}^{-} \text {in } \Omega_{-} \\
\operatorname{div} \mathbf{u}^{+}=g^{+} \text {in } \Omega_{+}^{h}, & \operatorname{div} \mathbf{u}^{-}=g^{-} \text {in } \Omega_{-},
\end{array}
$$

with coupling conditions

$$
\mathbf{u}^{+}=\mathbf{u}^{-}, \quad \frac{\partial}{\partial x_{3}} \mathbf{u}^{+}-\frac{\partial}{\partial x_{3}} \mathbf{u}^{-}=b \quad \text { on } \Sigma_{0},
$$

and unchanged boundary conditions on the remaining part of the boundary. Here $\mathbf{f}, g, b$ are given. Regularity on each subdomain follows from [15] by using technics for regularity of the transmission problems (see [6], proposition II.8.9).

Finally we can give rigorous justification of Remark 4.1

Corollary 4.4. $F \in C^{1}\left(\mathbb{R}_{+}\right)$, where function $F$ is defined by (2.1).

Proof. This corollary is direct consequence of Lemma 4.3 and the implicit function theorem.

Now we turn to proof of the following theorem:

THEOREM 4.5. $\mathbf{S}^{\prime}$ is either regular operator or the Fredholm operator with index 0.

Proof. We give the proof of this theorem in the series of lemmas.

Lemma 4.6. For every solution $\left(\mathbf{u}_{0}, p_{0}, h_{0}\right)$ of problem (4.2) there exists unique $\left(\mathbf{U}_{J}, P_{J}\right)$ such that $\overline{\mathbf{S}}^{\prime}\left(\mathbf{u}_{0}, p_{0}, h_{0}\right)\left(\mathbf{U}_{J}, P_{J}, 1\right)=0$, where $\overline{\mathbf{S}}=\left(S_{1}, S_{2}\right)$. Furthermore, for every $\mathbf{f} \in \mathcal{W} \times \mathcal{Q}$ there exists unique $\left(\mathbf{U}_{f}, P_{f}\right)$ such that $\overline{\mathbf{S}}^{\prime}\left(\mathbf{u}_{0}, p_{0}, h_{0}\right)\left(\mathbf{U}_{f}, P_{f}, 0\right)=\mathbf{f}$.

Proof. Proof follows directly from (4.3) and Lemma 4.3.

Let $\left(\mathbf{u}_{0}, p_{0}, h_{0}\right)$ be a solution of problem (4.2) and $\mathbf{f} \in \mathcal{W} \times \mathcal{Q}$. We define functions

$$
\mathbf{u}_{f, h}=h \mathbf{U}_{J}+\mathbf{U}_{f} \quad \text { and } \quad p_{f, h}=h P_{J}+P_{f}, \quad \mathbf{f} \in \mathcal{W} \times \mathcal{Q}, h \in \mathbb{R}_{+} .
$$

Here $\left(\mathbf{U}_{J}, P_{J}\right)$ and $\left(\mathbf{U}_{f}, P_{f}\right)$ are as in the above lemma. Notice that for every $h \in \mathbb{R}_{+}$the following equalities hold

$$
\begin{aligned}
\overline{\mathbf{S}}^{\prime}\left(\mathbf{u}_{0}, p_{0}, h_{0}\right)\left(\mathbf{u}_{f, h}, p_{f, h}, h\right)= & \overline{\mathbf{S}}^{\prime}\left(\mathbf{u}_{0}, p_{0}, h_{0}\right)\left(\mathbf{U}_{\mathbf{f}}, p_{f}, 0\right) \\
& +h \overline{\mathbf{S}}^{\prime}\left(\mathbf{u}_{0}, p_{0}, h_{0}\right)\left(\mathbf{U}_{J}, P_{J}, 1\right)=\mathbf{f} .
\end{aligned}
$$


Using $(4.4)_{3}$ we have:

$$
\begin{aligned}
& \mathbf{S}_{3}^{\prime}\left(\mathbf{u}_{0}, p_{0}, h_{0}\right)\left(\mathbf{u}_{f, h}, p_{f, h}, h\right) \\
& =h \int_{\Sigma}\left(\cos \alpha P_{J}-\mu \sin \alpha\left(\frac{1}{h_{0}} \partial_{x_{3}}\left(U_{J}\right)_{x_{1}}-\frac{1}{h_{0}^{2}} \partial_{x_{3}}\left(u_{0}\right)_{x_{1}}\right)\right) \\
& \quad+\cos \alpha \int_{\Sigma} P_{f}-\mu \sin \alpha \int_{\Sigma} \frac{1}{h_{0}} \partial_{x_{3}}\left(U_{f}\right)_{x_{1}} .
\end{aligned}
$$

LEMMA 4.7. Operator $\mathbf{S}^{\prime}\left(\mathbf{u}_{0}, p_{0}, h_{0}\right)$ is regular if and only if the following condition is satisfied:

$$
\int_{\Sigma}\left(\cos \alpha P_{J}-\mu \sin \alpha\left(\frac{1}{h_{0}} \partial_{x_{3}}\left(U_{J}\right)_{x_{1}}-\frac{1}{h_{0}^{2}} \partial_{x_{3}}\left(u_{0}\right)_{x_{1}}\right)\right) \neq 0
$$

Proof. First, we denote expression on the left-hand side of (4.5) by $D_{J}$. Let us take $(\mathbf{f}, s) \in \mathcal{W} \times \mathcal{Q} \times \mathbb{R}$ and take

$$
h=\frac{1}{D_{J}}\left(s-\left(\cos \alpha \int_{\Sigma} P_{f}-\mu \sin \alpha \int_{\Sigma} \frac{1}{h_{0}} \partial_{x_{3}}\left(U_{f}\right)_{x_{1}}\right)\right) .
$$

Then we have

$$
\mathbf{S}^{\prime}\left(\mathbf{u}_{0}, p_{0}, h_{0}\right)\left(\mathbf{u}_{f, h}, p_{f, h}, h\right)=(\mathbf{f}, s),
$$

and therefore we have proved surjectivity. For fixed $h$ we know from (4.3) and Lemma 4.3 that $\overline{\mathbf{S}}^{\prime}\left(\mathbf{u}_{0}, p_{0}, h_{0}\right)$ is regular and therefore all solutions of the the linearized problem are of the form $\left(\mathbf{u}_{f, h}, p_{f, h}\right)$. Since we can calculate $h$ by formula for given $s$, we have proved injectivity also. If condition (4.5) is not satisfied we do not have surjectivity because $(\mathbf{f}, s)$ is not in the image of $\mathbf{S}^{\prime}$ if $s \neq \cos \alpha \int_{\Sigma} P_{f}-\mu \sin \alpha \int_{\Sigma} \frac{1}{h_{0}} \partial_{x_{3}}\left(U_{f}\right)_{x_{1}}$.

Now it only remains to prove the last lemma:

LEMma 4.8. If condition (4.5) does not hold, then $\mathbf{S}^{\prime}\left(\mathbf{u}_{0}, p_{0}, h_{0}\right)$ is a Fredholm operator with index 0.

Proof. The triple $\left(\mathbf{U}_{\mathbf{J}}, P_{J}, 1\right)$ is obviously in kernel of $\mathbf{S}^{\prime}\left(\mathbf{u}_{0}, p_{0}, h_{0}\right)$. The fact that $\mathbf{U}_{0}=0$ and $P_{0}=0$ ensures that the basis of the kernel consists of this element only, where $\mathbf{U}_{0}$ and $P_{0}$ are functions introduced in Lemma 4.6 for $\mathbf{f}=0$.

Let us now consider image of $\mathbf{S}^{\prime}\left(\mathbf{u}_{0}, p_{0}, h_{0}\right)$. In this case we can also conclude that all solutions of equation $\overline{\mathbf{S}}^{\prime}\left(\mathbf{u}_{0}, p_{0}, h_{0}\right)(\mathbf{u}, p, h)=f$ are of the form $\left(\mathbf{u}_{f, h}, p_{f, h}, h\right)$. From condition (4.5) we have $\mathbf{S}_{3}^{\prime}\left(\mathbf{u}_{0}, p_{0}, h_{0}\right)\left(\mathbf{u}_{f, h}, p_{f, h}, h\right)$ $=C(f)$. Hence, the image of $\mathbf{S}^{\prime}$ is $\{(f, C(f)), f \in \mathcal{W} \times \mathcal{Q}\}$. Therefore we can conclude that co-kernel is $\{0\} \times\{0\} \times \mathbb{R}$.

Now everything is set for stating and proving the main theorem of this section. 
THEOREM 4.9. There exists $\alpha$ and $P_{0}$ with corresponding stationary state $\left(\mathbf{u}_{0}, p_{0}, h_{0}\right) \in \mathcal{V} \times \mathcal{Q} \times \mathbb{R}_{+}$in which we have a turning point. More precisely, $\left(\mathbf{u}_{0}, p_{0}, h_{0}\right)$ is a solution of problem (3.2) and all solutions of this problem in some neighborhood belong to some curve $(X(s), P(s))$ in $\left(\mathcal{V} \times \mathcal{Q} \times \mathbb{R}_{+}\right) \times(-a, a)$ with $X(0)=\left(\mathbf{u}_{0}, p_{0}, h_{0}\right)$ and $P(0)=P_{0}, a \in \mathbb{R}_{+}$. Furthermore, tangent at $(X(0), P(0))$ is $(V, 0)$ and $P$ does not have a saddle point at 0 .

Proof. Let us consider the operator $\mathbf{S}$ as a function of parameter $P_{0}$; taking its derivative we get

$$
D_{P_{0}} \mathbf{S}\left(\mathbf{u}_{0}, p_{0}, h_{0} ; P_{0}, \alpha\right)=(0,0,-1) \notin \operatorname{Im}^{\prime}\left(\mathbf{u}_{0}, p_{0}, h_{0} ; P_{0}, \alpha\right) .
$$

Now because of Theorem I.4.1 and Corollary I.4.2 from [13], we conclude that the point $\left(\mathbf{u}_{0}, p_{0}, h_{0}\right)$ is saddle nod bifurcation or turning point. It only remains to prove that there exists a stationary state $\left(\mathbf{u}_{0}, p_{0}, h_{0}\right)$ such that condition (4.5) is not true, i.e., the Frechet derivative of $\mathbf{S}$ is not regular. From previous considerations we know that this is equivalent to solving a problem:

find $(\mathbf{u}, p) \in \mathcal{V} \times \mathcal{Q}$ such that

$$
\begin{aligned}
& \overline{\mathcal{S}}\left(h_{0}\right)(\mathbf{u}, p)=-\overline{\mathcal{S}}^{\prime}\left(h_{0}\right)\left(\mathbf{u}_{0}, p_{0}\right), \\
& \int_{\Sigma}\left(\cos \alpha p-\mu \sin \alpha\left(\frac{1}{h_{0}} \partial_{x_{3}} u_{x_{1}}-\frac{1}{h_{0}^{2}} \partial_{x_{3}}\left(u_{0}\right)_{x_{1}}\right)\right)=0 .
\end{aligned}
$$

We solve this problem by using similar techniques as in solving the original problem (3.2). First using a change of variables we can return to the original domain $\Omega_{h_{0}}^{\alpha}$ and get an original-like problem, but this time with the right hand side dominated by hydrostatic pressure (which is included in $p_{0}$ ) as $h_{0} \rightarrow \infty$. Therefore we have

$$
\int_{\Sigma}\left(\cos \alpha p-\mu \sin \alpha\left(\frac{1}{h_{0}} \partial_{x_{3}} u_{x_{1}}-\frac{1}{h_{0}^{2}} \partial_{x_{3}}\left(u_{0}\right)_{x_{1}}\right)\right)<0
$$

for $h_{0}$ large enough, so is enough to find some point at which

$$
\int_{\Sigma}\left(\cos \alpha p-\mu \sin \alpha\left(\frac{1}{h_{0}} \partial_{x_{3}} u_{x_{1}}-\frac{1}{h_{0}^{2}} \partial_{x_{3}}\left(u_{0}\right)_{x_{1}}\right)\right)>0
$$

holds. Furthermore, we notice that solution depends continuously on parameter $\alpha$. Now it only remains to check the symmetry properties of $\overline{\mathcal{S}}^{\prime}\left(h_{0}\right)$ :

$$
\begin{aligned}
& \overline{\mathcal{S}}_{x_{1}}^{\prime}\left(h_{0} ; \alpha\right)\left(u_{0 x_{1}}^{\alpha}, u_{0 x^{\prime}}^{\alpha}, q_{0}^{\alpha}\right)=\overline{\mathcal{S}}_{x_{1}}^{\prime}\left(h_{0} ;-\alpha\right)\left(\bar{u}_{0 x_{1}}^{-\alpha},-\bar{u}_{0 x^{\prime}}^{-\alpha},-\bar{q}_{0}^{-\alpha}\right), \\
& \overline{\mathcal{S}}_{x^{\prime}}^{\prime}\left(h_{0} ; \alpha\right)\left(u_{0 x_{1}}^{\alpha}, u_{0 x^{\prime}}^{\alpha}, q_{0}^{\alpha}\right)=-\overline{\mathcal{S}}_{x^{\prime}}^{\prime}\left(h_{0} ;-\alpha\right)\left(\bar{u}_{0 x_{1}}^{-\alpha},-\bar{u}_{0 x^{\prime}}^{-\alpha},-\bar{q}_{0}^{-\alpha}\right), \\
& \overline{\mathcal{S}}_{2}^{\prime}\left(h_{0} ; \alpha\right)\left(u_{0 x_{1}}^{\alpha}, u_{0 x^{\prime}}^{\alpha}, q_{0}^{\alpha}\right)=-\overline{\mathcal{S}}_{2}^{\prime}\left(h_{0} ;-\alpha\right)\left(\bar{u}_{0 x_{1}}^{-\alpha},-\bar{u}_{0 x^{\prime}}^{-\alpha},-\bar{q}_{0}^{-\alpha}\right) ;
\end{aligned}
$$

here $\bar{f}$ stands for a function defined by $\bar{f}\left(x_{1}, x^{\prime}\right)=f\left(-x_{1}, x^{\prime}\right)$ for a function $f$ defined on $\Omega$. We can use analogous argument as in Remark 4.1 to prove 
existence of a point with desired property. From the same Remark 4.1 it follows also that we do not have saddle point.

COROLlary 4.10. With notations of the preceding theorems we have

$$
\left(F^{\alpha}\right)^{\prime}(h)=\int_{\Sigma}\left(\cos \alpha P_{J}-\mu \sin \alpha\left(\frac{1}{h_{0}} \partial_{x_{3}}\left(U_{J}\right)_{x_{1}}-\frac{1}{h_{0}^{2}} \partial_{x_{3}}\left(u_{0}\right)_{x_{1}}\right)\right), \quad h>0 .
$$

REMARK 4.11. This theorem also gives effective way of verifying whether some point is turning point or not as well as for the numerical search of that point. We could also use smooth change of variables to avoid non-standard function spaces, but then we would get more complicated formula for the linearized problem. Finally, in the proof of theorem 4.9 we need the symmetry properties:

$$
\begin{aligned}
& \left(\beta_{h}^{\alpha}\right)_{x_{1}}\left(x_{1}, x^{\prime}\right)=-\left(\beta_{h}^{-\alpha}\right)_{x_{1}}\left(-x_{1}, x^{\prime}\right) \\
& \left(\beta_{h}^{\alpha}\right)_{x^{\prime}}\left(x_{1}, x^{\prime}\right)=\left(\beta_{h}^{-\alpha}\right)_{x^{\prime}}\left(-x_{1}, x^{\prime}\right) .
\end{aligned}
$$

REMARK 4.12. If the horizontal pipe is long enough, then the symmetry conditions can be omitted. Namely, we can symmetrize it by extending shorter end of the pipe and prescribe suitable boundary conditions on the new artificial boundary. From asymptotic analysis (see for example [14]) we know which pressure we have to prescribe. Solution of our new problem is close to the solution of original problem. Similar analysis can be done for infinite horizontal pipe and other artificial boundary conditions.

4.2. Comments on the Navier-Stokes case. In the preceding section we have proved existence of a stationary state $\left(\mathbf{u}_{0}, p_{0}, h_{0}\right)$ (in case of small data) of our problem in the Navier-Stokes case (Theorem 3.5). By using regularity results in polyhedral domains due to Maz'ya and Rossmann ([15]) and using linearization technique we have proved non-uniqueness of solution and the bifurcation result (Theorem 4.9).

One should notice that three facts were essential in the proof. First, regularity result for the Stokes equation in polyhedral domains. In the recent paper by Maz'ya and Rossmann ([16]) the analogous result is proved for the Navier-Stokes equation.

Second fact is calculation of the Frechet derivative $\mathbf{S}^{\prime}$ and regularity of $\overline{\mathcal{S}}(h)$ for every fixed $h$ (Lemma 4.3). In the Navier-Stokes case formula (4.3) is not valid any more because we have additional non-linearity in $\mathbf{u}$, but only in the first component $\mathbf{S}_{1}$. In that case we have the following formula for the Frechet derivative of that component:

$$
\begin{aligned}
\mathbf{S}_{1}^{\prime}\left(\mathbf{u}_{0}, p_{0}, h_{0}\right)(\mathbf{u}, p, h)= & \mathcal{S}\left(h_{0}\right)(\mathbf{u}, p)+S_{L}\left(\mathbf{u}_{0}, p_{0}, h_{0}\right)(\mathbf{u}, p) \\
& +h\left(\mathcal{S}^{\prime}\left(h_{0}\right)\left(\mathbf{u}_{0}, p_{0}\right)+\mathcal{N}\left(h_{0}\right)\left(\mathbf{u}_{\mathbf{0}}, p_{0}\right)\right),
\end{aligned}
$$


where $\mathcal{S}$ is the same as before and

$$
\begin{aligned}
& \left\langle S_{L}\left(\mathbf{u}_{0}, p_{0}, h_{0}\right)(\mathbf{u}, p), \mathbf{v}\right\rangle \\
& \quad=h_{0} \int_{\Omega_{+}}\left(\left(\nabla^{h_{0}, \alpha} \mathbf{u}_{0}\right) \mathbf{u}+\left(\nabla^{h_{0}, \alpha} \mathbf{u}\right) \mathbf{u}_{0}\right) \cdot \mathbf{v}+\int_{\Omega_{-}}\left(\left(\nabla \mathbf{u}_{0}\right) \mathbf{u}+(\nabla \mathbf{u}) \mathbf{u}_{0}\right) \cdot \mathbf{v} \\
& \left\langle\mathcal{N}\left(h_{0}\right)\left(\mathbf{u}_{0}, p_{0}\right), \mathbf{v}\right\rangle=\int_{\Omega_{+}}\left(\left(\nabla^{h_{0}, \alpha} \mathbf{u}_{0}\right) \mathbf{u}_{0}-\frac{1}{h_{0}}\left(\left(\begin{array}{c}
\mathbf{0} \\
\partial_{x_{3}}
\end{array}\right) \mathbf{u}_{0}\right) \mathbf{u}_{0}\right) \cdot \mathbf{v} .
\end{aligned}
$$

However, this linearization is known since by transforming equations back to original domain we get linearized Navier-Stokes equation around $\left(\mathbf{u}_{0}, p_{0}\right)$. Then we can prove analogue of Lemma 4.3 in the Navier-Stokes case by using well known for the linearized Navier-Stokes equation (see [11]) and the same function spaces as in the Stokes case.

Third essential fact in the proof of Theorem 4.9 was symmetry properties of solution. In the Navier-Stokes case we do not have these symmetry properties, so we can not prove existence of a turning point. However, if a turning point exists, we know that it is of the same form as the one from the Stokes case which is described in Theorem 4.9 Furthermore, if we take data sufficiently small such that solution is dominated by the linear part, we can get existence of a turning point by approximating solution of the NavierStokes problem by solution of the Stokes problem.

\section{NumERICAL EXPERIMENTS}

All numerical experiments are done in 2D Stokes case using FreeFem++.

EXAMPLE 5.1. In this example we compute numerical values of function $F^{\frac{\pi}{4}}$ with $g=0$. For every fixed $h$ we solve Stokes system in $\Omega_{h}^{\frac{\pi}{4}}$ and then compute $F^{\frac{\pi}{4}}(h)$. We take $h$ between 0 and 4.2 with step 0.14 . Other parameters are: $l=5, P_{p / k}= \pm 5, d_{1}=1.6, d=1.6$ and $\mu=1$, where $d$ is diameter of the piston. For velocity $P 2$ elements are used and for pressure $P 1$.

In figure 2 graph of $F^{\frac{\pi}{4}}$ is given. On this example we can see the nonuniqueness of the stationary state since function $F^{\frac{\pi}{4}}$ is clearly non-monotone. Furthermore, we can see that function $F^{\frac{\pi}{4}}$ is asymptotically approaching some constant as we have proved earlier. We can also notice that this constant is greater than 0 which means that in this case piston rises higher then in symmetric case which is physically expected since "vertical" pipe is along the flow.

EXAMPLE 5.2. In this example we compute $F^{\prime}(h)$ with same parameters as in the previous example.

EXAMPLE 5.3. As we have proven for every given geometry and data there exists constant $C_{\alpha}$ such that $\lim _{h \rightarrow \infty} F^{\alpha}(h)=\cos \alpha C_{\alpha}$. The goal of 


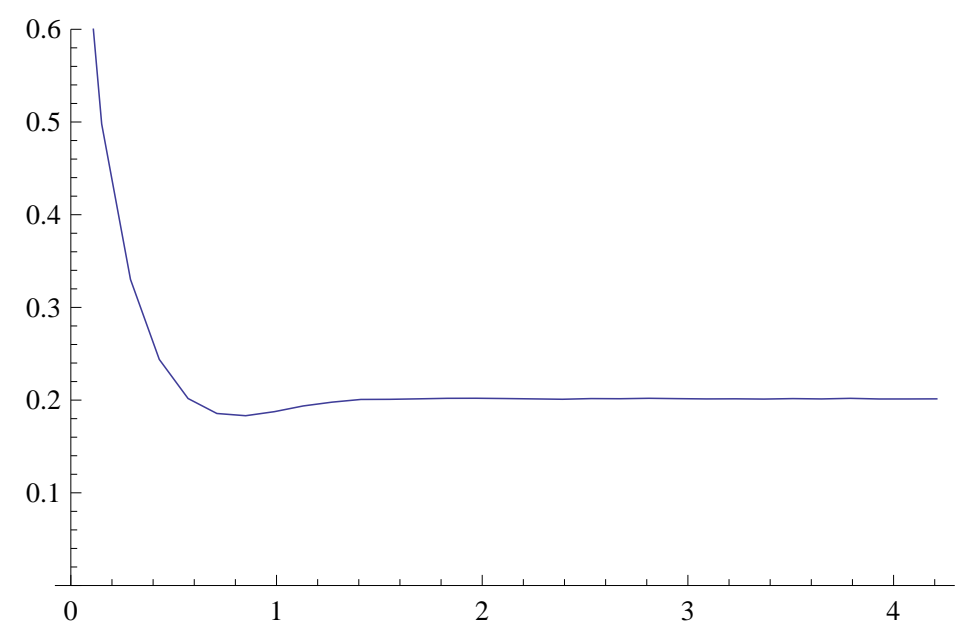

Figure 2. Graph of function $F$

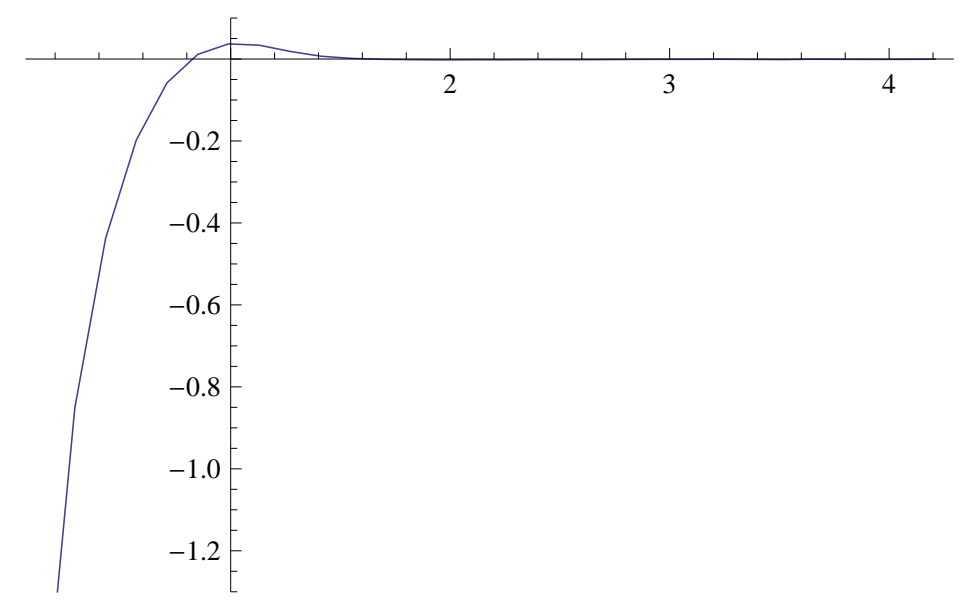

FIGURE 3. Graph of $F^{\prime}$

this example is to analyze dependence of constants $C_{\alpha}$ on parameter $\alpha$. We take all parameters the same as in previous example, $h=5$ (which is large enough) and vary parameter $\alpha$ between $\frac{-3}{8} \pi$ and $\frac{3}{8} \pi$ with step $\frac{\pi}{24}$ and compute $F^{\alpha}(5)$. 


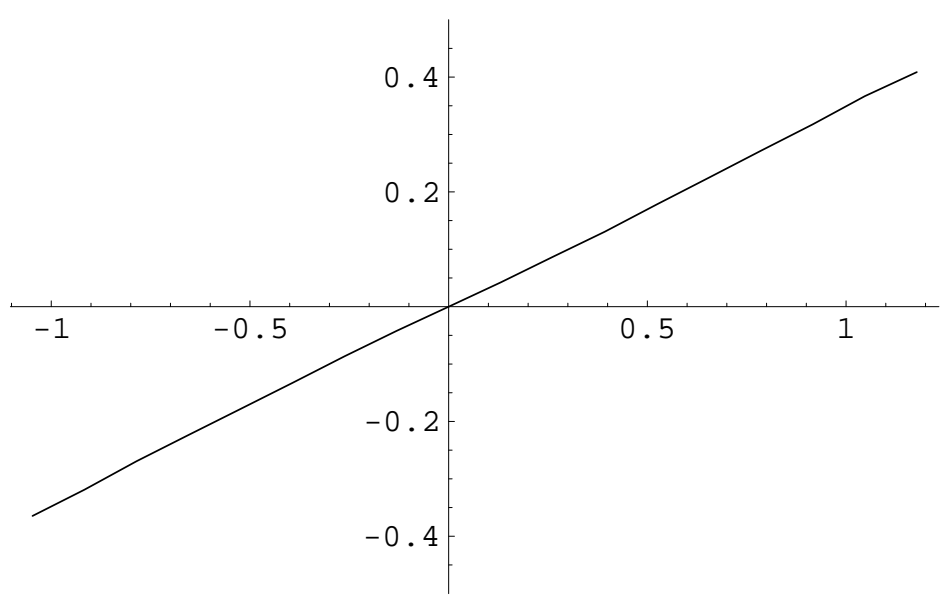

Figure 4. Dependence of $C_{\alpha}$ on $\alpha$

ACKNowledGements.

We are grateful to Prof. I. Aganović for valuable discussions regarding formulation of the problem.

\section{REFERENCES}

[1] J. M. Bernard, Non-standard Stokes and Navier-Stokes problems: existence and regularity in stationary case, Math. Methods Appl. Sci. 25 (2002), 627-661.

[2] S. Blazy, S. Nazarov and M. Specovius-Neugebauer, Artificial boundary conditions of pressure type for viscous flows in a system of pipes, J. Math. Fluid Mech. 9 (2007), $1-33$.

[3] C. Conca, F. Murat and O. Pironneau, The Stokes and Navier-Stokes equations with boundary conditions involving the pressure, Japan. J. Math. (N.S.) 20 (1994), 279318.

[4] C. Conca, J. San Martín H. and M. Tucsnak. Motion of a rigid body in a viscous fluid, C. R. Acad. Sci. Paris Sér. I Math. 328 (1999), 473-478.

[5] B. D'Acunto and S. Rionero, A note on the existence and uniqueness of solutions to a free piston problem, Rend. Accad. Sci. Fis. Mat. Napoli (4) 66 (1999), 75-84.

[6] R. Dautray and J.-L. Lions. Mathematical analysis and numerical methods for science and technology. Vol. 1, Physical origins and classical methods, With the collaboration of Philippe Bénilan, Michel Cessenat, André Gervat, Alain Kavenoky and Hélène Lanchon, Translated from the French by Ian N. Sneddon, With a preface by Jean Teillac, Springer-Verlag, Berlin, 1990.

[7] B. Desjardins and M. J. Esteban, On weak solutions for fluid-rigid structure interaction: compressible and incompressible models, Comm. Partial Differential Equations 25 (2000), 1399-1413.

[8] G. P. Galdi, An introduction to the mathematical theory of the Navier-Stokes equations. Vol. I. Linearized steady problems, Springer-Verlag, New York, 1994.

[9] G. P. Galdi, An introduction to the mathematical theory of the Navier-Stokes equations. Vol. II. Nonlinear steady problems, Springer-Verlag, New York, 1994. 
[10] G. P. Galdi, Mathematical problems in classical and non-Newtonian fluid mechanics, in: Hemodynamical flows, Birkhäuser, Basel, 2008, 121-273.

[11] V. Girault and P.-A. Raviart, Finite element methods for Navier-Stokes equations. Theory and algorithms, Springer-Verlag, Berlin, 1986.

[12] M. Hillairet and D. Serre, Chute stationnaire d'un solide dans un fluide visqueux incompressible le long d'un plan incliné, Ann. Inst. H. Poincaré Anal. Non Linéaire 20 (2003), 779-803.

[13] H. Kielhöfer, Bifurcation theory. An introduction with applications to PDEs, Springer-Verlag, New York, 2004.

[14] E. Marušić-Paloka, Rigorous justification of the Kirchhoff law for junction of thin pipes filled with viscous fluid, Asymptot. Anal. 33 (2003), 51-66.

[15] V. Maz'ya and J. Rossmann, $L_{p}$ estimates of solutions to mixed boundary value problems for the Stokes system in polyhedral domains, Math. Nachr. 280 (2007), 751-793.

[16] V. Maz'ya and J. Rossmann, Mixed boundary value problems for the stationary Navier-Stokes system in polyhedral domains, Arch. Ration. Mech. Anal. 194 (2009), 669-712.

[17] J. Nečas, Les méthodes directes en théorie des équations elliptiques, Masson et Cie, Éditeurs, Paris, 1967.

[18] V. G. Osmolovskiĭ, Linear and nonlinear perturbations of the operator div. Translated from the 1995 Russian original by Tamara Rozhkovskaya, American Mathematical Society, Providence, 1997.

[19] S. Takeno, Free piston problem for isentropic gas dynamics, Japan J. Indust. Appl. Math. 12 (1995), 163-194.

[20] R. Temam, Navier-Stokes equations. Theory and numerical analysis, North-Holland Publishing Co., Amsterdam, 1977.

B. Muha

Department of Mathematics

University of Zagreb

10000 Zagreb

Croatia

E-mail: borism@math.hr

Z. Tutek

Department of Mathematics

University of Zagreb

10000 Zagreb

Croatia

E-mail: tutek@math.hr

Received: 29.7.2011. 